\title{
End the Routine Shackling of Incarcerated Inpatients
}

\author{
Marc Robinson, $\mathrm{MD}^{1 *}$, Kristen Lavere, MD ${ }^{1}$, Esmaeil Porsa, MD, MBA, MPH ${ }^{1,2}$
}

1'Department of Medicine, Baylor College of Medicine, Houston, Texas; ${ }^{2}$ Department of Internal Medicine, McGovern Medical School at UTHealth, Houston, Texas; ${ }^{3}$ Department of Internal Medicine, Baylor College of Medicine, Houston, Texas.

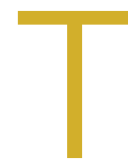

he police shooting of Jacob Blake, an unarmed Wisconsin man, during an arrest in August 2020, led to more protests in a summer filled with calls against the unequal application of police force. Outrage grew as it was revealed that Blake, paralyzed from his waist down and not yet convicted of a crime, was still handcuffed to his hospital bed while receiving treatment. ${ }^{1}$ To many this seemed unusually cruel, but to those tasked with caring for incarcerated patients, it is all too familiar. Given the high rates of incarceration in the United States and the increased medical needs of this population, caring for those in custody is unavoidable for many physicians and hospitals. Though safety should be paramount, the universal application of metal handcuffs or leg cuffs by law enforcement officials, a process known as shackling, can lead to a variety of harms and should be abandoned.

\section{BACKGROUND}

The United States incarcerates more individuals both in total numbers and per capita than any other country in the world. This is currently believed to be more than two million people on any given day or more than 650 persons per 100,000 population. ${ }^{2}$ Incarceration occurs in jails, which are locally run facilities holding individuals on short sentences or those not yet convicted who are unable to afford bail before their trials (pretrial), or prisons, which are state and federally run facilities that house those with long sentences. When an incarcerated person experiences a medical emergency requiring hospitalization, they are either treated in the correctional facility or transferred to a local hospital for a higher level of care. Some hospitals are equipped with security measures similar to those of a correctional facility, with secure floors or wings dedicated solely to the care of the incarcerated. Secure units are more commonly seen in hospitals associated with prisons rather than local jails. Other hospitals house incarcerated patients in the same rooms as the public population, and thus movement is restricted by other means. ${ }^{3}$ Most commonly, this is done with a hard metal shackle resembling a handcuff with one end attached to the leg or wrist and the other end attached to the bed. Some agencies require more restraints, often requiring

*Corresponding Author: Marc Robinson, MD; Email: Marc.Robinson@bcm.edu; Telephone: 713-873-3560; Twitter: @MarcRobinsonMD.

Published online first May 19, 2021.

Received: August 19, 2020; Revised: November 30, 2020;

Accepted: December 1, 2020

๑ 2021 Society of Hospital Medicine DOI 10.12788/jhm.3581 the use of wrist cuffs and leg cuffs concurrently for the entire duration of a patient's hospitalization. ${ }^{4}$ In our experience, agencies apply these restraints universally, regardless of age, illness, mobility, or pretrial status.

Restraint practices are rooted in a concern for practitioner and public safety and bear merit. A patient from a correctional facility is usually guarded by just one officer in lieu of the multiple security measures at a jail or prison facility. Nonsecured hospitals have become sites of multiple escapes by incarcerated inpatients, given the lack of secured doors and the multiple movements during the admission and discharge processes. ${ }^{5}$ Furthermore, violence against hospital staff is now a focus issue in many hospitals and is no longer accepted as just "part of the job." In several high-profile incidents, incarcerated inpatients have harmed staff, including one at our own institution, when an incarcerated patient held a makeshift weapon to a student's throat. ${ }^{6}$

\section{LEGAL CHALLENGES}

The use of shackles during hospital visits has been challenged in US courts and routinely upheld. In one case, an incarcerated patient with renal failure received injuries after his leg edema was so severe that "at one point the shackles themselves were barely visible." ${ }^{77}$ Though he was injured, the shackles were determined to have served a penological purpose outside of punishment, such as preventing escape, and the injuries were the result of the patient's guards not following protocol. British courts have taken a different stance, ruling for an incarcerated patient who challenged the use of cuffs during three outpatient appointments and one inpatient admission. ${ }^{8}$ While the cuffs in the outpatient setting were deemed acceptable (as they were removed during the medical visit itself), they remained during the duration of the inpatient stay. This was deemed in violation of Title I/Article 3 of the Charter of Fundamental Rights of the European Union, Dignity/The right to integrity of the person. One area in US healthcare where shackling has been roundly condemned is the peripartum shackling of pregnant women. Though courts have had a mixed record to challenges, activism and advocacy have led to the banning of the practice in 23 states, though in most states significant exemptions exist. ${ }^{9}$ Through the First Step Act of 2018, the federal government banned peripartum shackling for all federal prisoners, but as most incarcerations are under state or local control, a considerable number of incarcerated pregnant women can legally be shackled during their deliveries.

\section{RISKS OF SHACKLING}

Legal and safety concerns aside, the shackling of incarcerated patients carries enormous risk. The use of medical restraints in hos- 
pitals has decreased over the past few decades, given their proven harms in increasing falls, exacerbating delirium, and increasing the risk of in-hospital death. ${ }^{10}$ There is no reason to believe that trading a soft medical restraint for a metal leg or wrist cuff would not confer the same risk. Additionally, metal law enforcement cuffs are not designed with patient safety in mind and have been known to cause specific nerve injuries, or handcuff neuropathy. This can occur when placement is too tight or when a patient struggles against them, as could happen with an agitated or delirious patient. The bar for removal, even briefly for an exam, is also much higher than that of a medical restraint, leading to a greater likelihood that certain aspects of the physical exam, such as gait or strength assessment, may not be adequately performed. In one small survey, British physicians reported often performing an exam while the patient was cuffed and with a guard in the room, despite country guidelines against both practices. ${ }^{11}$

Additionally, marginalized communities are disproportionately incarcerated and have a fraught and tenuous relationship with the healthcare system. Black patients routinely report greater mistrust than White patients in the outcomes of care and the motivations of physicians, in large part due to past and current discrimination and the medical community's history of experimentation. ${ }^{12}$ A shackled patient may view a treating physician and hospital as complicit with the practice, rather than seeing the practice as something outside of their control. If a patient's sole interaction with inpatient medicine involves shackling, it risks damaging whatever fragile physician-patient relationship may exist and could delay or limit care even further.

While the universal application of metal handcuffs or leg cuffs ensures low rates of escape or attacks on workers, it does so at the expense of vulnerable individuals. We have cared for an incarcerated elderly woman arrested for multiple traffic violations, a man with severe autism who slipped through the cracks of mental health diversion protocols and ended up in jail, and an arrested delirious man with severe alcohol withdrawal, all shackled with hard shackles on the wrists, legs, or in the final case, both. Safety and the rights of the vulnerable are not mutually exclusive, and we feel the following measures can protect both.

\section{A WAY FORWARD}

First, the universal application of shackles in the hospitalized incarcerated patient should end. If no alternative security measures are available for high-risk patients, correctional facilities must document their necessity as physicians and nurses are required to do for medical restraints. Hospitals should have processes in place for providers who feel unsafe with an unshackled patient or think a patient is unnecessarily shackled, and collegial discussions about shackling with law enforcement should be the norm. If safe to do so, shackles should routinely be removed for physical exams without question. Since law enforcement officials, rather than the hospitals, make the rules for shackling, this will take some degree of physician and administrative advocacy at the hospital level and legislative advocacy at the local and state levels.

Second, vulnerable populations, such as the elderly, those experiencing a mental health crisis, or others at risk for in-hospital delirium, should never be restrained with hard law enforcement cuffs. Restraint procedures should follow standard medical restraint procedures, and soft restraints should be used if at all possible. Given the high rates of psychiatric illness amongst the incarcerated and the role jails play in filling gaps in psychiatric care, medical admissions for those with mental illness are not rare occasions.

Finally, hospitals routinely taking care of an incarcerated population should seek to build secure units, a move that would dramatically reduce the need for shackling. In several cities, the primary referral hospitals for some of the largest jails in the country do not have units with the proper security to allow for freedom of movement, and thus, shackling persists. Creating secure units will take significant investment on the part of hospital and local authorities, but there is potential for decreasing costs due to consolidating supervision, which would lead to better patient outcomes given the above risks.

Advocating for the health of the incarcerated, even those who have not yet been convicted, is typically not a high priority for the general public. As inpatient physicians, we see the impact universal shackling has on some of our most vulnerable patients and should be their voice where they have none. Advocating for and implementing the above procedures will be a step toward improving patient care while maintaining safety.

Disclosures: The authors have no conflicts to disclose.

\section{References}

1. Proctor C. Jacob Blake handcuffed to hospital bed, father says. Chicago Sun-Times. Updated August 27, 2020. Accessed December 29, 2020. chicago.suntimes.com/2020/8/27/21404463/jacob-blake-father-kenosha -police-shooting-hospital-bed-handcuffs

2. Maruschak LM, Minton TD. Correctional populations in the United States, 2017-2018. Bureau of Justice Statistics. August 2020. Accessed September 30, 2020. https://www.bjs.gov/content/pub/pdf/cpus1718.pdf

3. Huh K, Boucher A, Fehr S, McGaffey F, McKillop M, Schiff M. State prisons and the delivery of hospital care: how states set up and finance off-site care for incarcerated individuals. The Pew Charitable Trusts. July 2018. Accessed September 30, 2020. https://www.pewtrusts.org/-/media/assets/2018/07 /prisons-and-hospital-care_report.pdf

4. Haber LA, Erickson HP, Ranji SR, Ortiz GM, Pratt LA. Acute care for patients who are incarcerated: a review. JAMA Intern Med. 2019;179(11):1561-1567. https://doi.org/10.1001/jamainternmed.2019.3881

5. Mikow-Porto VA, Smith TA. The IHSSF 2011 Prisoner Escape Study. J Healthc Prot Manage. 2011;27(2):38-58.

6. Lezon D, Blakinger K. Inmate shot by deputy after holding medical student at Ben Taub. Houston Chronicle. October 6, 2016. Accessed December 29, 2020. https://www.chron.com/news/houston-texas/article/Deputy-shoots -suspect-at-Ben-Taub-hopsital-9873972.php

7. Yearwood LT. Pregnant and shackled: why inmates are still giving birth cuffed and bound. The Guardian. January 24, 2020. Accessed December 29, 2020. theguardian.com/us-news/2020/jan/24/shackled-pregnant-women -prisoners-birth

8. Haslar v Megerman, 104 F.3d 178 (8th Cir. 1997)

9. FGP $\vee$ Serco Plc and SSHD, EWHC 1804 (Admin) (2012).

10. Cleary K, Prescott $K$. The use of physical restraints in acute and longterm care: an updated review of the evidence, regulations, ethics, and legality. J Acute Care Phys Ther. 2015;6(1):8-15. https://doi.org/10.1097 /JAT.0000000000000005

11. Tuite H, Browne K, O'Neill D. Prisoners in general hospitals: doctors' attitudes and practice. BMJ. 2006;332(7540):548-549. https://doi.org/10.1136 /bmj.332.7540.548-b

12. LaVeist TA, Nickerson KJ, Bowie JV. Attitudes about racism, medical mistrust, and satisfaction with care among African American and white cardiac patients. Med Care Res Rev. 2000;57(Suppl 1):146-161. https://doi .org/10.1177/1077558700057001S07 